\title{
Intelligence on the Web and e-Inclusion
}

\author{
Laura Burzagli and Francesco Gabbanini \\ Institute of Applied Physics "Nello Carrara" - Italian National Research Council \\ Via Madonna del Piano 10, 50019 Sesto Fiorentino, Florence, Italy \\ \{L.Burzagli, F. Gabbanini\}@ifac.cnr. it
}

\begin{abstract}
Within the context of Web, the word intelligence is often connected with the visions of Semantic Web and Web 2.0. One of the main characteristic of Semantic Web lies in the fact that information is annotated with metadata and this gives the opportunity of organizing knowledge, extracting new knowledge and performing some basic operations like query answering or inference reasoning. Following this argument, the advent of the Semantic Web is often claimed to bring about substantial progress in Web accessibility (which is part of the e-Inclusion concept). Web 2.0 sites, favoring massive information sharing, could as well be of great importance for e-Inclusion, enabling new forms of social interaction, collective intelligence and new patterns of interpersonal communication. Benefits could be substantial also for people with activity limitations. The paper tries to highlight the possible roles and convergence of Web 2.0 and Semantic Web in favoring e-Inclusion. It highlights the fact that examples of applications of these concepts to the e-Inclusion domain are few and limited to the e-Accessibility field.
\end{abstract}

Keywords: e-Inclusion, Web 2.0, Semantic Web.

\section{Introduction}

Due to the evolution and increased complexity of the Web, intelligence is becoming a challenging functionality in the Web itself, and a number of forms in which it can manifest itself have been identified, such as the Semantic Web and Web 2.0.

Enhancements that these forms of intelligence might bring in access to information and interpersonal communication could have a positive impact in the field of e-Inclusion.

The term e-Inclusion is here considered in its widest definition, both as a support to accessibility of Information and Communication Technology and as a support to the daily activities of people, according to the European Union Riga declaration ${ }^{1}$, point 4:

"e-Inclusion" means both inclusive ICT and the use of ICT to achieve wider inclusion objectives. It focuses on participation of all individuals and communities in all aspects of the information society. E-Inclusion policy, therefore, aims at reducing

\footnotetext{
${ }^{1}$ See http://ec.europa.eu/information_society/events/ict_riga_2006/doc/declaration_riga.pdf, last visited on $2 / 27 / 2009$.
} 
gaps in ICT usage and promoting the use of ICT to overcome exclusion, and improve economic performance, employment opportunities, quality of life, social participation and cohesion.

The analysis in this paper introduces Web 2.0 and Semantic Web, tries to highlight their possible convergence and to summarize their role in favoring e-Inclusion. The discussion points out the fact that, up to now, few examples of applications of Semantic Web and Web 2.0 to the e-Inclusion domain exist, and they are mostly limited to the e-Accessibility field. An example of one of such applications is presented to stimulate the discussion.

\section{Aspects of Intelligence on the Web}

Web technologies have been constantly evolving, and also the way in which the Web is used and perceived by its users is evolving. As observed in [1], from the browsing of some pages containing text or images, going through the connection of pages, the Web has become an interactive ubiquitous information system that leverages the wisdom of many users and makes it possible to reuse data through mashups. From the perspective of users this structure offers information, services and powerful search engines to find them. Users can take advantage of an environment useful to people for research, learning, commerce, socialization, communication and entertainment. In order to fully exploit the potential of today's Web, benefits would come from the introduction of intelligence embedded on the system, to handle complex scenarios.

Intelligence on the web can be considered according to several different perspectives.

In the complex and varied Web world, a noteworthy phenomenon is assuming a great importance: Web 2.0. According to the opinion of several experts, this phenomenon is not based only on technological innovations, but draws its peculiarity from social aspects, from the cooperation between users and the wide variety of web contents which are directly generated from users. This combination of technological and human factors could represent a valuable support also in the e-Inclusion field.

However, at scientific level, the more advanced representation of intelligence on Web seems to be the Semantic Web, the revolution for the web proposed by Tim Berners-Lee almost 10 years ago [2]. If referred to e-Inclusion the concept seems to offer a number of features that not only can improve web accessibility and overcome several limitations which are present in today's Web, but also can lead to the creation of new services which can be useful for a wide variety of users (see [3], [4]).

An interesting aspect to note is that Semantic Web and Web 2.0 are not in contrast, but it has become clear to many experts (see [5], [6]) that they are natural complements of each other, because Semantic Web can give a formal representation of human meaning, while contents can be built and maintained using the techniques and data generation capabilities that are typical of Web 2.0.

\subsection{Web 2.0: Social and Technological Aspects}

Although there is not full agreement on a definition, Web 2.0 (also called the wisdom Web, people-centric Web, participative Web, see [7], [8]) is perceived as a second phase in the Web's evolution, in which web based services have the characteristic of 
aiming to facilitate collaboration and sharing between users, letting them engage in an interactive and collaborative manner, giving more emphasis to social interaction and collective intelligence. It is a fact that the term does not refer to an update to Web technical specifications, but to changes in the way software developers use known web technologies and in the way end-users use the internet as a platform.

A number of researches attribute to this phenomenon an element of intelligence (see [8], [9], [7]). Intelligence originates from interaction among users, when this interaction happens by means of the use of Internet, and differs from intelligence seen as a result of software routines implementing Artificial Intelligence procedures.

In the literature, someone describe this aspect with the terms collected intelligence (i.e. the value of user contributions is in their being collected together), finding them more appropriate than collective intelligence (i.e. characterized by the emergence of truly new levels of understanding) (for example, see [10]).

Accessibility Issues in Web 2.0. From the technological point of view, many (but not all) Web 2.0 applications are supported by a series of new generation web based technologies that have existed since the early days of the web, but are now used in such a way to exploit user-generated content, resource sharing and interactivity in a more sophisticated and powerful way (see [11]), giving rise to the so called Rich Internet Applications (RIA). Techniques such as AJAX have evolved that have the potential to improve the user-experience in browser-based applications. The main impact on accessibility comes from dynamic and incremental updates in web pages. From the one side, these may come unexpected to users, who may not notice that a part of the page has changed. From a second side it is to be noted that problems with asynchronous updates may be fatal for users relying on Assistive Technology (AT): in fact, updates can occur on a different area of the page than where the user is currently interacting and AT's could fail notifying users that something on the page has changed. Issues concerning accessibility in RIA's are being faced by the WAI-ARIA ${ }^{2}$ of the W3C, which has formulated a series of best practices for rich internet applications design. WAI-ARIA markup presents a solution to making these applications accessible.

An interesting analysis of technologies to enable a more accessible Web 2.0, discussed in [12], points out that, basically, ARIA is built upon Semantic Web concepts in that it defines so called "live regions" that allow, adding semantic annotations to the HTML and XHTML markup in order to better define the role of user interface components. This feature can be used, for example, to enable assistive technologies to give an appropriate representation of user interfaces. For example a browser can interpret the additional semantic data and provide it to the assistive technology via the accessibility Application Programming Interface of the platform, which already implements mechanisms to describe user interface controls.

Thiessen and Chen in [13] present a chat example that shows ARIA live regions in action and demonstrates several of their limitations.

Web 2.0 perspectives for e-Inclusion. As a result of a first survey, it appears that the role of Web 2.0 with respect to e-Inclusion (considered from the perspective of its general definition) has not yet been object of interest in the scientific community.

\footnotetext{
${ }^{2}$ See http://www.w3.org/WAI/intro/aria.php. Last visited on 2/27/2009.
} 
Up to now, Web 2.0 has almost exclusively been considered useful in particular fields of applications such as leisure, travel or e-commerce. The availability of a wide corpus of collective intelligence could give Web 2.0 an important role also in the field of e-Inclusion, where interaction among users has always been considered (see [12] as an example) to offer valuable support in helping people to overcome limitations (either physical or cultural), even if only a limited number of examples have been presented that follow this direction, yet.

The new forms of social interaction and collective intelligence brought by Web 2.0 could enable new patterns of interpersonal communication for all users. Benefits could be substantial also for people with activity limitations. For example, through Web 2.0 sites motor impaired users could share their experiences about accessible accommodations and paths in towns: value to the application could increase as more users use it, putting their knowledge at the disposal of other users.

An example of a service that exploits the capabilities of Web 2.0 and Semantic Web in a sub-domain of e-Inclusion, that is e-Accessibility, is described in Section 4.

It is to be noted, moreover, that the interaction techniques that are typical of this Web 2.0 (although a number of problems related to their accessibility are emerging and are being handled by WAI) can represent a useful help for some group of users, especially for people with cognitive disabilities because of the possibility to implementing contextual help, tailoring interfaces to meet users' experiences and because of the fact that techniques exist to learn users' preferences (see [5]).

\subsection{Semantic Web}

The World Wide Web is a container of knowledge as well as a mean to exchange information between peoples and let them communicate.

As of today, even if pages are generated by CMS and stored in databases, they are presented in the form of pages written in mark-up languages such as HTML and XHTML. Thus, web contents are mostly structured to be readable by humans and not by machines.

The aim of the Semantic Web initiative (originated by Tim Berners-Lee and now being developed within the World Wide Web Consortium ${ }^{3}$ ) is to represent web contents in a manner that is more easily processable by machines and to set up tools to take advantage of this representation. The initiative seeks to provide a common framework that allows data to be shared and reused across application, enterprise, and community boundaries. In this way, according to the Semantic Web vision, a person, or a machine, should be capable to start browsing information on the web and then move through a set of information sources which are connected not by wires and links, but by being "about the same thing" (see [14] for example).

The Semantic Web is based on the concept of ontology: an ontology is used to describe formally a domain of discourse and consists of a list of terms and the relationships between these terms. Metadata, organized with ontologies, are used to identify information in web sources and logic is used for processing retrieved information and uncover knowledge and relationships found in ontologies.

\footnotetext{
${ }^{3}$ See http://www.w3.org/2001/sw/. Last visited on 2/27/2009.
} 
It is to be noted that the Semantic Web does not aim to exhibit a human-level intelligence, as the one envisioned by Artificial Intelligence (AI). Though it builds on the work carried out in AI, the Semantic Web stack (made up with RDF, RDFSchema, Ontology vocabularies, Rules, Logic and Proof) aims at building intelligent agents, in the sense that they are capable of uncovering unexpected relationships between concepts.

Semantic Web Perspectives for e-Inclusion. Discussions on the role of Semantic Web and e-Inclusion are mainly focused on aspects related to e-Accessibility.

It is highlighted by several authors that the Semantic Web is not an area that is very well explored for supporting Web accessibility (see [5], [15]). However, it is also generally acknowledged that developments connected to the Semantic Web can provide a valuable contribution to creating accessible content, especially if taken together with Web 2.0.

One of the main characteristic of Semantic data is that it can be modeless: it is not already deliberately constructed as a page. Following this argument, the advent of the Semantic Web is often claimed to bring about substantial progress in Web accessibility and presentation on mobile devices (which are part of the e-Inclusion concept), as it facilitates providing alternatives for content and form for documents (see, e.g., [16]).

Harper and Bechhofer, in [4], observe that semantic information built into general purpose Web pages could enable substantial improvements of accessibility of web pages. In fact, information is often rendered on web pages in an order defined by the designer and not in the order required by the user and web pages may bear implicit meaning that is connected to how to the information presented visually, while, for example, the same meaning cannot be interpreted by visually impaired persons that are forced to interact with systems in a serial manner.

The availability of semantics would be of great value for Assistive Technology, which relies on semantic understanding to provide access to the user interfaces.

Moreover, this could also provide benefits in that it could enable automatic reformulation and rearrangement of contents, based on metadata, in view of their fruition by people with different preferences and needs, in different contexts of use.

\section{Convergence Between Web 2.0 and Semantic Web}

So far Web 2.0 and Semantic Web, which are considered two visions of the intelligence on the web, have been considered as completely different approaches to the future web. However, recently (see [6] for example), a number of authors have started to consider a possible convergence between them, merging their most successful aspects. From one side, the richness of Web 2.0 lies in its social dimension, and is characterized by an easy exchange of information in wide communities (social network) and in the collection of large amount of information, even if this is often unstructured. From the other side, the strength of Semantic Web is in its capability of interlinking and reusing structured information, but it needs data to be aggregated and recombined. In other words there is the need to merge human participation with well structured information. 


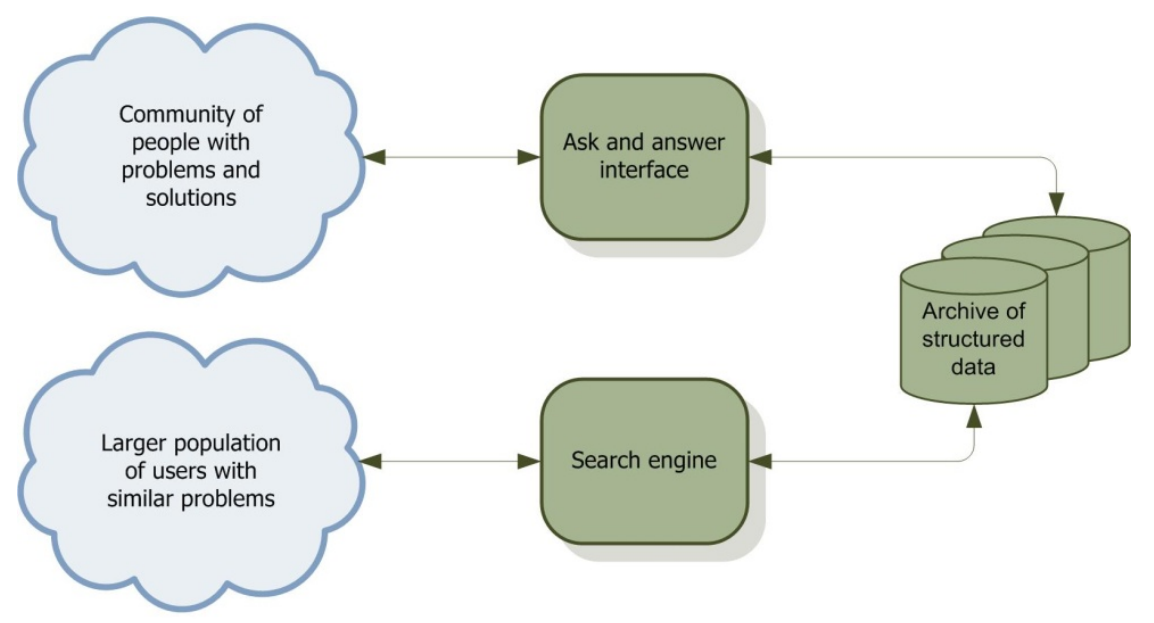

Human users

Machine processes

Fig. 1. The "collective knowledge system" general scheme

Two main solutions appear in literature. The first is related ([5]) to the creation of services and tool that are able to automatically analyze web pages and discover semantics, thus allowing structuring users generated content.

A different solution (see [10]) is represented by systems that are defined by Gruber as "collective knowledge systems", in which existing semantic structures are used to organize large amounts of knowledge generated by users (see Fig. 1).

These systems are made up with a social network, supported by computing and communication technology, in which self-service problem solving discussions take place and where people pose problems and others reply with answers; a search engine to find questions and answers; users helping the system learning about which query/document pairs were effective at addressing their problems.

According to Gruber [10], the role of Semantic Web is firstly seen in adding value to user data by adding structured data, related to the content of the user contributions in a form that enables more powerful computation. Secondly, Semantic Web technologies can enable data sharing and computation across independent, heterogeneous Social Web applications, whereas, up to now, these data are presently confined in a given application.

\section{Existing Applications}

An existing example of the collective knowledge systems outlined in Section 3 is presented in the next section.

The example is given by the IBM Social Accessibility Project. Though it regards eAccessibility, it is taken as a model to highlight potential benefits that could come from the exploitation of the convergence of Semantic Web and Web 2.0, in the wider domain of e-Inclusion. 


\subsection{The IBM Social Accessibility Project}

The Social Accessibility Project (see screenshot in Fig. 2) has been set up by IBM to improve Web accessibility by using the power of communities.

It is a service whose goal is to make Web pages more accessible to people with disabilities, taking advantage from users' input and leveraging on the power of the open community while not changing any existing content.

The system allows users encountering Web access problems to immediately report them to the Social Accessibility server.

Volunteers (called supporters) can be quickly notified and can easily respond by creating and publishing the requested accessibility metadata, which will help other users who encounter the same problems.

Specifically, supporters are able to discuss solutions among themselves through Web applications on the server and create a set of metadata to solve the problem; they then submit it to the server.

When the user visits the page again, the page is automatically fixed and any user who installs a suitable software extension can access the accessible version of the page.

This project delineates an interesting possible convergence between Web 2.0 and the Semantic Web because it takes advantage of a social network that discusses problems and tries to provide solutions in a collaborative manner.

There is a potentially continuous interaction between users and supporters to discuss solutions and consider comments.

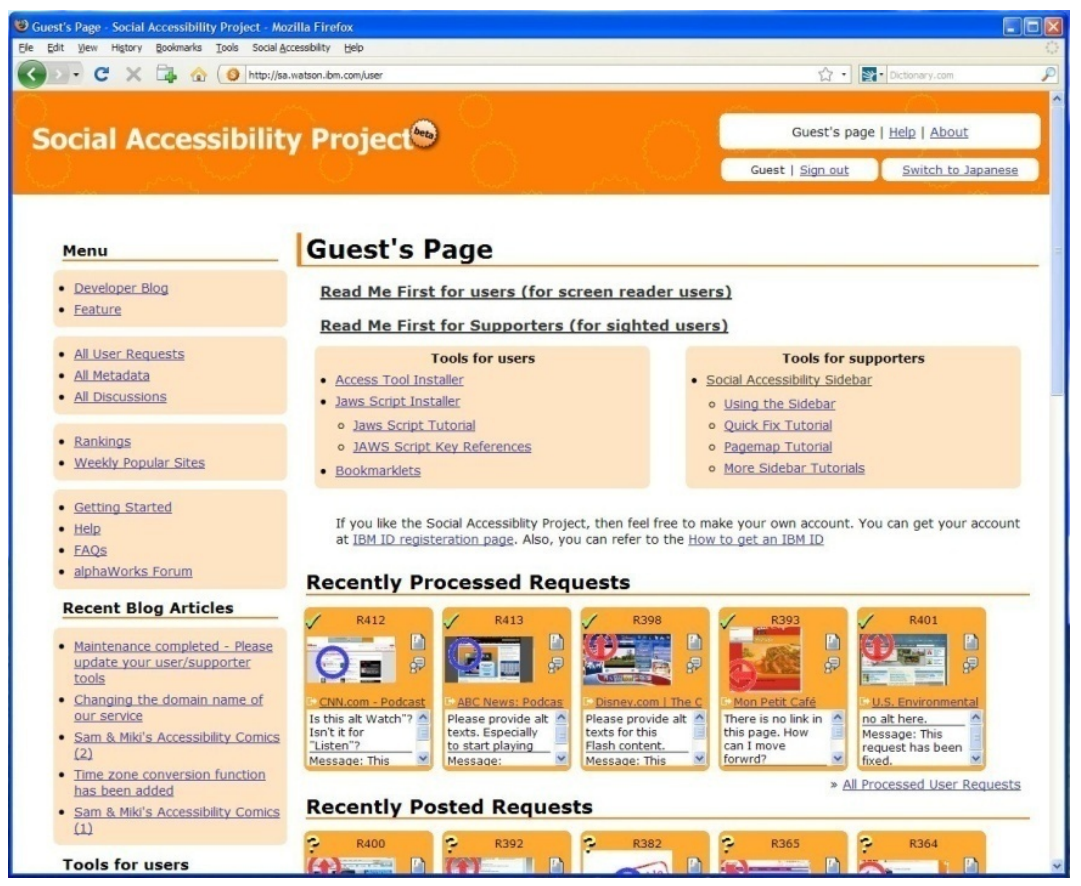

Fig. 2. Screenshot of the gust page of the IBM Social Accessibility Project 
Users can also create metadata: for example when a user finds an important position in a page, the position can be submitted as a "landmark" for other users. This process ends up with the creation of metadata that help identifying and overcoming access problems.

More details on the service can be found at the address http://sa.watson.ibm.com/.

\section{Conclusions}

The paper presents Semantic Web and Web 2.0 as two different approaches for the distribution of intelligence on the web.

Their characteristics are presented and a summary of potential benefits coming from the application of Web 2.0 and Semantic Web vision to the e-Inclusion field is discussed.

A class of applications (named "collective knowledge systems"), which in the future could serve as an example for exploiting the convergence of Semantic Web and Web 2.0 and which could have a positive impact on e-Inclusion is presented. An example referred to the improvement of accessibility of the Web is described.

It is to be noted that the Web has certainly evolved from a collection of hypertext pages to an interactive ubiquitous information system that leverages the wisdom of many users to provide knowledge on virtually all fields. Web 2.0 has indeed had a relevant impact on the evolution of the Web, whereas the contribution of Semantic Web, though presently less visible, is expected to have an influence at longer terms.

However, the evolution of Web and the uptake of Web 2.0 and Semantic Web seem to have had, up to now, a limited impact on e-Inclusion and they have been mostly studied in relation to the impact on a sub-domain of e-Inclusion, that is, eAccessibility. In fact, in the literature that was examined during the authors' work, very few specific references of Web 2.0 to e-Inclusion were found, with the exception of a number of works dealing with problems that Web 2.0 technology can bring to the accessibility of web sites (which, indeed, is an aspect of e-Inclusion) or some minor applications built to provide accessible interfaces to some Web 2.0 applications like YouTube $^{4}$ and SlideShare ${ }^{5}$.

The IBM Social Accessibility Project, though focused again on accessibility, seems to represent a valid example of a new perspective in that it exemplifies a class of applications that take advantage of the power of Web 2.0 and Semantic Web and could have a positive impact on e-Inclusion.

Acknowledgements. The contribution of Pier Luigi Emiliani in the development of the ideas presented in the paper is warmly acknowledged.

\section{References}

1. Kelly, K.: Four stages in the internet of things (November 2007),

http://www.kk.org/thetechnium/archives/2007/11/

four_stages_in.php (last visited on 2/27/2009)

\footnotetext{
${ }^{4}$ See http://icant.co.uk/easy-youtube/, last visited on 2/26/2009

${ }^{5}$ See http://icant.co.uk/easy-slideshare/about/index.html, last visited on 2/26/2009
} 
2. Berners-Lee, T., Hendler, J., Lassila, O.: The semantic web. Scientific American 284(5), 34-43 (2001)

3. Kouroupetroglou, C., Salampasis, M., Manitsaris, A.: A semantic-web based framework for developing applications to improve accessibility in the WWW. In: W4A: Proceedings of the 2006 international cross-disciplinary workshop on Web accessibility (W4A), pp. 98-108. ACM Press, New York (2006)

4. Harper, S., Bechhofer, S.: Semantic triage for increased web accessibility. IBM Systems Journal 44(3), 637-648 (2005)

5. Cooper, M.: Accessibility of emerging rich web technologies: Web 2.0 and the Semantic Web. In: W4A 2007: Proceedings of the 2007 international cross-disciplinary conference on Web accessibility (W4A), pp. 93-98. ACM Press, New York (2007)

6. Heath, T., Motta, E.: Ease of interaction plus ease of integration: Combining web2.0 and the semantic web in a reviewing site. Web Semantics 6(1), 76-83 (2008)

7. Murugesan, S.: Understanding web 2.0. IT Professional 9(4), 34-41 (2007)

8. O'Reilly, T.: What is web 2.0? Design Patterns and Business Models for the Next Generation of Software (September 2005),

http://www.oreilly.com/pub/a/oreilly/tim/news/2005/09/30/

what-is-web-20.html

9. Lin, K.J.: Building Web 2.0. Computer 40(5), 101-102 (2007)

10. Gruber, T.: Collective knowledge systems: Where the Social Web meets the Semantic Web. Journal of Web Semantics 6(1), 4-13 (2008)

11. Knights, M.: Web 2.0. Communications Engineer 5(1), 30-35 (2007)

12. Gibson, B.: Enabling an accessible Web 2.0. In: W4A 2007: Proceedings of the 2007 international cross-disciplinary conference on Web accessibility (W4A), pp. 1-6. ACM Press, New York (2007)

13. Thiessen, P., Chen, C.: Ajax live regions: chat as a case example. In: W4A 2007: Proceedings of the 2007 international cross-disciplinary conference on Web accessibility (W4A), pp. 7-14. ACM Press, New York (2007)

14. Antoniou, G., Van Harmelen, F.: A Semantic Web Primer, 2nd edn. MIT Press, Cambridge (2008)

15. Yesilada, Y., Harper, S.: Web 2.0 and the semantic web: hindrance or opportunity? In: W4a - international cross-disciplinary conference on web accessibility 2007. SIGACCESS Accessibility and Computing, vol. (90), pp. 19-31 (2008)

16. Seeman, L.: The semantic web, web accessibility, and device independence. In: Proceedings of the 2004 international Cross-Disciplinary Workshop on Web Accessibility (W4A), vol. 63, pp. 67-73. ACM Press, New York (2004) 